\title{
A WAY TO GENERATE SINGULAR MATRIX
}

\author{
MARIJA MITEVA ${ }^{1}$, LIMONKA KOCEVA LAZAROVA, NATASA STOJKOVIK, \\ AND ALEKSANDRA STOJANOVA
}

\begin{abstract}
In this work we consider some matrix products when the resulting matrix is singular matrix. The main result is a way to obtain singular matrix by multiplication of two random matrices with specific form: when we multiply $n \times k$ matrix with $k \times n$ one, for $n>k$, we always obtain singular $n \times n$ matrix as result.
\end{abstract}

\section{INTRODUCTION}

In the mathematics and in many other sciences, especially programming, the matrices are so important and applicable. In [1] are presented applicationoriented techniques with matrices in modern linear algebra with the emphasis on data mining and pattern recognition. The matrix theory is widely used in many different areas in the engineering like: wireless communications, signal processing, control etc. The authors in [2] give an overview about applications of the matrix theory in the wireless communications and the signal processing. They discuss that the concept of singular value decompositions is the most important in multiple antennas-based communication systems. Also, in that paper are given more applications of the matrices in signal processing and signal detection. The big relationship between the matrices and the images is explained too. In [3] it is shown that the matrices, particularly singular matrices over

${ }^{1}$ corresponding author

2010 Mathematics Subject Classification. 15A29, $15 B 52$.

Key words and phrases. matrix, matrix multiplication, determinant of matrix, singular matrix. 
finite fields have huge application in cryptography. In that paper the authors using singular matrices determine a key exchange method in which two users over an insecure channel want to agree a secret key.

Singular matrix $A$ is matrix which determinant is equal to 0 , i.e. $\operatorname{det} A=0$. The singular matrix does not have inverse matrix. Otherwise, the matrix is called non-singular matrix. In [4] is set the question: How rare are the singular matrices? The author of that paper noticed that in elementary linear algebra many examples of singular matrices can be found. But, how many of them occur naturally? Are they given randomly? In most of the examples, if the students encounter singular matrix they can easily transform it into a non-singular matrix (to change its $n-1$ rank into a full rank). Because of the rarity of the singular matrix it is enough to change only one of the entries of the singular matrix.

Many authors in their work consider problems related to singular matrices. It is shown in [5] that a probability randomly chosen square matrix to be singular is equal to 0 . From topological point of view in [6] is proved that the set of singular $n \times n$ matrices over the real numbers $S(\mathbf{R})$ is closed and nowhere dense in the set of all $n \times n$ matrices over the real numbers $\mathbf{M}_{n}(\mathbf{R})$. In [7], the author considers the linear singular space, i.e. the space in which each matrix is singular matrix. The author presents many examples in that paper for that subspace of the space of all $n \times n$ matrices over the real numbers $\mathbf{M}_{n}(\mathbf{R})$, because characterization of such subspaces would solve many problems in the combinatorics and computational algebra. Many useful properties of the singular matrices are presented and proved in [8].

The author in [9] noted that not only the non-singular matrices, but also some singular matrices form a group under the usual law of composition or multiplication. It is shown that not every singular matrix belongs to such group. The author provide classification of all matrices as to their group-membership and exact relationship to groups. Also, the conditions for group membership are determined.

Not only the matrices over some field of numbers are applicable. In the last period many papers are devoted to the matrices in which the entries are random variables. For these random matrices in many papers is considered the probability that such random matrix is singular. In [10] the authors estimated the probability that random $n \times n \pm 1$ - matrix is singular. They have showed that the probability the random $n \times n \pm 1$ - matrix is singular is $\mathbf{P}_{n}<(1-\varepsilon)^{n}$, where $\varepsilon$ 
is constant. Similar estimations for the probability that the random $n \times n$ matrix, where all the entries are discrete random variables is singular are made in [11]. The authors in that paper, have shown that if for all the entries of the matrix for a constant $0<p<1$ and a constant positive integer $r$ can be defined a property $p$ bounded of exponent $r$, then the probability that the random $n \times n$ matrix is singular is at most $\left(p^{\frac{1}{r}}+o(1)\right)^{n}$, where $o(1)$ tends to 0 , when $n$ tends to $\infty$.

We are going to show in the next section how one can obtain $n \times n$ singular matrix, multiplying two randomly chosen matrices with specific form.

We will use only basic (well-known) definitions and notations from matrix theory. We can only mention that with $[A]_{n \times k}$ we will denote $n \times k$ matrix, i.e. matrix with $n$ rows and $k$ columns.

\section{RESUlts: A WAY TO GENERATE SINGULAR MATRIX}

In this section we will prove firstly in separate theorems that multiplying $n \times 1$ matrix with $1 \times n$ one (for $n>1$ ), then $n \times 2$ matrix with $2 \times n$ one (for $n>2$ ) and $n \times 3$ matrix with $3 \times n$ one (for $n>3$ ), we always obtain singular matrix as a result. After that, we will prove the general case, that multiplying $n \times k$ matrix with $k \times n$ one, for $n>k$, the product obtained is singular matrix.

We are proving the first three cases separately in order the reader to deal easily with some (maybe complicated) notations in the proof of the general case.

Theorem 2.1. Multiplying $n \times 1$ matrix $A$ with $1 \times n$ matrix $B$, for $n>1$, we always obtain as result singular $n \times n$ matrix $A B$.

Proof. This is trivial case. If we take $A=\left[\begin{array}{c}a_{1} \\ a_{2} \\ \ldots \\ a_{n}\end{array}\right]$ and $B=\left[\begin{array}{llll}b_{1} & b_{2} & \ldots & b_{n}\end{array}\right]$, the product $A B$ will be:

$$
A B=\left[\begin{array}{c}
a_{1} \\
a_{2} \\
\ldots \\
a_{n}
\end{array}\right] \cdot\left[\begin{array}{llll}
b_{1} & b_{2} & \ldots & b_{n}
\end{array}\right]=\left[\begin{array}{cccc}
a_{1} b_{1} & a_{1} b_{2} & \ldots & a_{1} b_{n} \\
a_{2} b_{1} & a_{2} b_{2} & \ldots & a_{2} b_{n} \\
\ldots & \ldots & \ldots & \ldots \\
a_{n} b_{1} & a_{n} b_{2} & \ldots & a_{n} b_{n}
\end{array}\right]
$$


which determinant is:

$$
\operatorname{det} A B=\left|\begin{array}{cccc}
a_{1} b_{1} & a_{1} b_{2} & \ldots & a_{1} b_{n} \\
a_{2} b_{1} & a_{2} b_{2} & \ldots & a_{2} b_{n} \\
\ldots & \ldots & \ldots & \ldots \\
a_{n} b_{1} & a_{n} b_{2} & \ldots & a_{n} b_{n}
\end{array}\right|=a_{1} a_{2} \ldots a_{n}\left|\begin{array}{cccc}
b_{1} & b_{2} & \ldots & b_{n} \\
b_{1} & b_{2} & \ldots & b_{n} \\
\ldots & \ldots & \ldots & \ldots \\
b_{1} & b_{2} & \ldots & b_{n}
\end{array}\right|=0
$$

This proves the theorem.

Theorem 2.2. Multiplying $n \times 2$ matrix with $2 \times n$ one, for $n>2$, we always obtain singular $n \times n$ matrix as result.

Proof. The proof will be done by induction.

1) We will prove first the case $n=3$. If we take $A=[A]_{3 \times 2}=\left[\begin{array}{ll}a & b \\ c & d \\ e & f\end{array}\right]$ and $B=[B]_{2 \times 3}=\left[\begin{array}{lll}x & y & z \\ s & k & u\end{array}\right]$, we will prove that the product $A B$ is singular $3 \times 3$ matrix:

$$
A B=\left[\begin{array}{ll}
a & b \\
c & d \\
e & f
\end{array}\right] \cdot\left[\begin{array}{ccc}
x & y & z \\
s & k & u
\end{array}\right]=\left[\begin{array}{ccc}
a x+b s & a y+b k & a z+b u \\
c x+d s & c y+d k & c z+d u \\
e x+f s & e y+f k & e z+f u
\end{array}\right]
$$

To calculate the determinant of this matrix, we will multiply the second and the third row with $\frac{1}{a} \cdot a$ and thus obtain:

$\operatorname{det} A B=\left|\begin{array}{ccc}a x+b s & a y+b k & a z+b u \\ c x+d s & c y+d k & c z+d u \\ e x+f s & e y+f k & e z+f u\end{array}\right|=\frac{1}{a^{2}}\left|\begin{array}{ccc}a x+b s & a y+b k & a z+b u \\ a c x+a d s & a c y+a d k & a c z+a d u \\ a e x+a f s & a e y+a f k & a e z+a f u\end{array}\right|$

We will add the first row multiplied by $(-c)$ to the second row and add the first row multiplied by $(-e)$ to the third row and obtain: 


$$
\begin{aligned}
& \operatorname{det} A B=\frac{1}{a^{2}}\left|\begin{array}{ccc}
a y+b k & a z+b u \\
a c x+a d s-a c x-c b s & a c y+a d k-a c y-c b k & a c z+a d u-a c z-c b u \\
a e x+a f s-a e x-e b s & a e y+a f k-a e y-e b k & a e z+a f u-a e z-e b u
\end{array}\right|= \\
& =\frac{1}{a^{2}}\left|\begin{array}{ccc}
a x+b s & a y+b k & a z+b u \\
a d s-c b s & a d k-c b k & a d u-c b u \\
a f s-e b s & a f k-e b k & a f u-e b u
\end{array}\right|=\frac{1}{a^{2}}\left|\begin{array}{ccc}
a x+b s & a y+b k & a z+b u \\
s(a d-c b) & k(a d-c b) & u(a d-c b) \\
s(a f-e b) & k(a f-e b) & u(a f-e b)
\end{array}\right|= \\
& =\frac{(a d-c b)(a f-e b)}{a^{2}}\left|\begin{array}{ccc}
a x+b s & a y+b k & a z+b u \\
s & k & u \\
s & k & u
\end{array}\right|=0
\end{aligned}
$$

Therefore, the product is singular matrix.

2) Now let we take $A=[A]_{4 \times 2}=\left[\begin{array}{cc}a & b \\ c & d \\ e & f \\ g & h\end{array}\right]$ and $B=[B]_{2 \times 4}=\left[\begin{array}{cccc}x & y & z & t \\ s & k & u & m\end{array}\right]$, then estimate $A B$ :

$$
A B=[A B]_{4 \times 4}=\left[\begin{array}{ll}
a & b \\
c & d \\
e & f \\
g & h
\end{array}\right] \cdot\left[\begin{array}{llll}
x & y & z & t \\
s & k & u & m
\end{array}\right]=\left[\begin{array}{llll}
a x+b s & a y+b k & a z+b u & a t+b m \\
c x+d s & c y+d k & c z+d u & c t+d m \\
e x+f s & e y+f k & e z+f u & e t+f m \\
g x+h s & g y+h k & g z+h u & g t+h m
\end{array}\right]
$$

Let us estimate the determinant of the resulting matrix, i.e. $\operatorname{det} A B$. If we use minor expansion formula, choosing the last row, we have:

$$
\begin{aligned}
& \operatorname{det} A B=-(g x+h s)\left|\begin{array}{ccc}
a y+b k & a z+b u & a t+b m \\
c y+d k & c z+d u & c t+d m \\
e y+f k & e z+f u & e t+f m
\end{array}\right|+(g y+h k)\left|\begin{array}{ccc}
a x+b s & a z+b u & a t+b m \\
c x+d s & c z+d u & c t+d m \\
e x+f s & e z+f u & e t+f m
\end{array}\right| \\
& -(g z+h u)\left|\begin{array}{lll}
a x+b s & a y+b k & a t+b m \\
c x+d s & c y+d k & c t+d m \\
e x+f s & e y+f k & e t+f m
\end{array}\right|+(g t+h m)\left|\begin{array}{lll}
a x+b s & a y+b k & a z+b u \\
c x+d s & c y+d k & c z+d u \\
e x+f s & e y+f k & e z+f u
\end{array}\right|
\end{aligned}
$$

If we look at the last minor, it is easy to notice that this minor can be consider as determinant of the matrix obtained by multiplying the matrices $[A]_{3 \times 2}$ and $[B]_{2 \times 3}$ in the previous case 1 ). Those matrices can be obtained from $A=[A]_{4 \times 2}$ and $B=[B]_{2 \times 4}$ given in this case 2), when erasing the last row in $A$ and the 
last column in $B$. Therefore, it is the determinant of matrix obtained as product of $3 \times 2$ and $2 \times 3$ matrix and we have already proved in the case 1 ) that this is singular matrix. It means that the last minor has value 0 .

If we look at the other three minors in the previous expansion, we can easily notice that each of them can be consider as determinant of $3 \times 3$ matrix obtained as result of multiplying $3 \times 2$ and $2 \times 3$ matrices: if we erase the last row in the matrix $A=[A]_{4 \times 2}$ and the first column in the matrix $B=[B]_{2 \times 4}$ given in this case 2), and then multiply thus obtained matrices, we will obtain as a product matrix which determinant is the first minor in the previous expansion. Erasing the last row in $A=[A]_{4 \times 2}$ and the second column in $B=[B]_{2 \times 4}$, then multiplying obtained matrices the result will be matrix with the same elements as elements in the second minor. In the same way the third minor can be consider as product of $3 \times 2$ and $2 \times 3$ matrices, erasing the last row in $A=[A]_{4 \times 2}$ and the third column in $B=[B]_{2 \times 4}$. According to the case 1) all these minors have value 0 , which means that $\operatorname{det} A B=0$. Therefore, multiplying $4 \times 2$ and $2 \times 4$ matrix, the result will always be singular matrix.

3) We suppose now that the Theorem 2.2 holds for the product of $(n-1) \times 2$ matrix with $2 \times(n-1)$ one.

4) If we take $A=[A]_{n \times 2}=\left[\begin{array}{cc}a_{11} & a_{12} \\ a_{21} & a_{22} \\ \ldots & \ldots \\ a_{n 1} & a_{n 2}\end{array}\right]$ and $B=[B]_{2 \times n}=\left[\begin{array}{cccc}b_{11} & b_{12} & \ldots & b_{1 n} \\ b_{21} & b_{22} & \ldots & b_{2 n}\end{array}\right]$ for the product $A B$ we have:

$$
A B=[A B]_{n \times n}=\left[\begin{array}{cccc}
a_{11} b_{11}+a_{12} b_{21} & a_{11} b_{12}+a_{12} b_{22} & \ldots & a_{11} b_{1 n}+a_{12} b_{2 n} \\
a_{21} b_{11}+a_{22} b_{21} & a_{21} b_{12}+a_{22} b_{22} & \ldots & a_{21} b_{1 n}+a_{22} b_{2 n} \\
\ldots & \ldots & \ldots & \ldots \\
a_{n 1} b_{11}+a_{n 2} b_{21} & a_{n 1} b_{12}+a_{n 2} b_{22} & \ldots & a_{n 1} b_{1 n}+a_{n 2} b_{2 n}
\end{array}\right]
$$

We can calculate the determinant of this matrix by minor expansion, choosing the last row. Each minor obtained can be consider as determinant of matrix that is product of $(n-1) \times 2$ matrix with $2 \times(n-1)$ one, similarly as in the case 2$)$. According to 3 ) all these minors have value 0 , which means that $\operatorname{det} A B$ in this 
case has value 0 , thus multiplying $n \times 2$ with $2 \times n$ matrix, the result is singular matrix.

Theorem 2.3. Multiplying matrix $A=[A]_{n \times 3}$ with matrix $B=[B]_{3 \times n}$, for $n>3$, we always obtain singular $A B=[A B]_{n \times n}$ matrix as result.

Proof. We will prove the theorem by induction.

1) First, we prove that multiplying $4 \times 3$ and $3 \times 4$ matrix, we obtain $4 \times 4$ singular matrix.

Let $A=[A]_{4 \times 3}=\left[\begin{array}{ccc}a & b & c \\ d & e & f \\ g & h & i \\ j & k & l\end{array}\right]$ and $B=[B]_{3 \times 4}=\left[\begin{array}{cccc}m & n & o & p \\ q & r & s & t \\ u & v & w & x\end{array}\right]$. With multiplication we obtain:

$$
A B=[A B]_{4 \times 4}=\left[\begin{array}{cccc}
a m+b q+c u & a n+b r+c v & a o+b s+c w & a p+b t+c x \\
d m+e q+f u & d n+e r+f v & d o+e s+f w & d p+e t+f x \\
g m+h q+i u & g n+h r+i v & g o+h s+i w & g p+h t+i x \\
j m+k q+l u & j n+k r+l v & j o+k s+l w & j p+k t+l x
\end{array}\right]
$$

Let we calculate the determinant of this matrix. First, we will multiply the second, the third and the last row with $\frac{1}{a} \cdot a$ and thus obtain:

$$
\begin{gathered}
\operatorname{det} A B=\left|\begin{array}{cccc}
a m+b q+c u & a n+b r+c v & a o+b s+c w & a p+b t+c x \\
d m+e q+f u & d n+e r+f v & d o+e s+f w & d p+e t+f x \\
g m+h q+i u & g n+h r+i v & g o+h s+i w & g p+h t+i x \\
j m+k q+l u & j n+k r+l v & j o+k s+l w & j p+k t+l x
\end{array}\right|= \\
=\frac{1}{a^{3}}\left|\begin{array}{cccc}
a m+b q+c u & a n+b r+c v & a o+b s+c w & a p+b t+c x \\
a d m+a e q+a f u & a d n+a e r+a f v & a d o+a e s+a f w & a d p+a e t+a f x \\
a g m+a h q+a i u & a g n+a h r+a i v & a g o+a h s+a i w & \text { agp }+ \text { aht }+ \text { aix } \\
\text { ajm +akq+alu } & \text { ajn+akr+alv } & \text { ajo+aks+alw } & \text { ajp }+ \text { akt }+ \text { alx }
\end{array}\right|
\end{gathered}
$$

We will add the first row multiplied with $(-d)$ to the second row, add the first row multiplied with $(-g)$ to the third row and add the first row multiplied with $(-j)$ to the last row and for $\operatorname{det} A B$ then obtain: 
$\frac{1}{a^{3}}\left|\begin{array}{cccc}a m+b q+c u & a n+b r+c v & a o+b s+c w & a p+b t+c x \\ q(a e-d b)+u(a f-d c) & r(a e-d b)+v(a f-d c) & s(a e-d b)+w(a f-d c) & t(a e-d b)+x(a f-d c) \\ q(a h-g b)+u(a i-g c) & r(a h-g b)+v(a i-g c) & s(a h-g b)+w(a i-g c) & t(a h-g b)+x(a i-g c) \\ q(a k-b j)+u(a l-j c) & r(a k-b j)+v(a l-j c) & s(a k-b j)+w(a l-j c) & t(a k-b j)+x(a l-j c)\end{array}\right|$

Considering $(a e-d b)$ as multiplier in the second row, $(a h-g b)$ as multiplier in the third row and considering $(a k-b j)$ as multiplier in the last row, we have:

$\operatorname{det} A B=\frac{(a e-d b)(a h-g b)(a k-b j)}{a^{3}}\left|\begin{array}{cccc}a m+b q+c u & a n+b r+c v & a o+b s+c w & a p+b t+c x \\ q+u \frac{a f-d c}{a e-d b} & r+v \frac{a f-d c}{a e-d b} & s+w \frac{a f-d c}{a e-d b} & t+x \frac{a f-d c}{a e-d b} \\ q+u \frac{a i-g c}{a h-g b} & r+v \frac{a i-g c}{a h-g b} & s+w \frac{a i-g c}{a h-g b} & t+x \frac{a i-g c}{a h-g b} \\ q+u \frac{a l-j c}{a k-b j} & r+v \frac{a l-j c}{a k-b j} & s+w \frac{a l-j c}{a k-b j} & t+x \frac{a l-j c}{a k-b j}\end{array}\right|$

Denoting $Q=\frac{(a e-d b)(a h-g b)(a k-b j)}{a^{3}}$ and adding the second row multiplied by $(-1)$ to the third and to the last row, we obtain:

$\operatorname{det} A B=Q\left|\begin{array}{cccc}a m+b q+c u & a n+b r+c v & a o+b s+c w & a p+b t+c x \\ q+u \frac{a f-d c}{a e-d b} & r+v \frac{a f-d c}{a e-d b} & s+w \frac{a f-d c}{a e-d b} & t+x \frac{a f-d c}{a e-d b} \\ u\left(\frac{a i-g c}{a h-g b}-\frac{a f-d c}{a e-d b}\right) & v\left(\frac{a i-g c}{a h-g b}-\frac{a f-d c}{a e-d b}\right) & w\left(\frac{a i-g c}{a h-g b}-\frac{a f-d c}{a e-d b}\right) & x\left(\frac{a i-g c}{a h-g b}-\frac{a f-d c}{a e-d b}\right) \\ u\left(\frac{a l-j c}{a k-b j}-\frac{a f-d c}{a e-d b}\right) & v\left(\frac{a l-j c}{a k-b j}-\frac{a f-d c}{a e-d b}\right) & w\left(\frac{a l-j c}{a k-b j}-\frac{a f-d c}{a e-d b}\right) & x\left(\frac{a l-j c}{a k-b j}-\frac{a f-d c}{a e-d b}\right)\end{array}\right|$

Denoting $M=\left(\frac{a i-g c}{a h-g b}-\frac{a f-d c}{a e-d b}\right)$ and $N=\left(\frac{a l-j c}{a k-b j}-\frac{a f-d c}{a e-d b}\right)$ in the last determinant, we have:

$$
\begin{aligned}
& \operatorname{det} A B=Q\left|\begin{array}{cccc}
a m+b q+c u & a n+b r+c v & a o+b s+c w & a p+b t+c x \\
q+u \frac{a f-d c}{a e-d b} & r+v \frac{a f-d c}{a e-d b} & s+w \frac{a f-d c}{a e-d b} & t+x \frac{a f-d c}{a e-d b} \\
u M & v M & w M & x M \\
u N & v N & w N & x N
\end{array}\right|= \\
& =Q M N\left|\begin{array}{cccc}
a m+b q+c u & a n+b r+c v & a o+b s+c w & a p+b t+c x \\
q+u \frac{a f-d c}{a e-d b} & r+v \frac{a f-d c}{a e-d b} & s+w \frac{a f-d c}{a e-d b} & t+x \frac{a f-d c}{a e-d b} \\
u & v & w & x \\
u & v & w & x
\end{array}\right|=0
\end{aligned}
$$

which proves that multiplying $4 \times 3$ with $3 \times 4$ matrix, we obtain $4 \times 4$ singular matrix. 
2) If we take $5 \times 3$ and $3 \times 5$ matrices: $A=\left[a_{i j}\right]_{5 \times 3}(i=\overline{1,5} ; j=\overline{1,3})$ and $B=\left[b_{k l}\right]_{3 \times 5}(k=\overline{1,3} ; l=\overline{1,5})$, multiplying $A$ with $B$ we have:

$$
A B=\left[\begin{array}{ccccc}
\sum a_{1 j} b_{j 1} & \sum a_{1 j} b_{j 2} & \sum a_{1 j} b_{j 3} & \sum a_{1 j} b_{j 4} & \sum a_{1 j} b_{j 5} \\
\sum a_{2 j} b_{j 1} & \sum a_{2 j} b_{j 2} & \sum a_{2 j} b_{j 3} & \sum a_{2 j} b_{j 4} & \sum a_{2 j} b_{j 5} \\
\sum a_{3 j} b_{j 1} & \sum a_{3 j} b_{j 2} & \sum a_{3 j} b_{j 3} & \sum a_{3 j} b_{j 4} & \sum a_{3 j} b_{j 5} \\
\sum a_{4 j} b_{j 1} & \sum a_{4 j} b_{j 2} & \sum a_{4 j} b_{j 3} & \sum a_{4 j} b_{j 4} & \sum a_{4 j} b_{j 5} \\
\sum a_{5 j} b_{j 1} & \sum a_{5 j} b_{j 2} & \sum a_{5 j} b_{j 3} & \sum a_{5 j} b_{j 4} & \sum a_{5 j} b_{j 5}
\end{array}\right]
$$

where $j=\overline{1,3}$ in each sum.

To calculate the determinant of this matrix we can use again the minor expansion formula choosing the last row. It is obvious that all minors in the expansion can be consider as determinant of matrix obtained with multiplication of $4 \times 3$ with $3 \times 4$ matrix (similar as in the case 2 in the previous theorem). According to the case 1 ) in this theorem, all the minors in the expansion will be 0 which means that multiplying $5 \times 3$ matrix with $3 \times 5$ one we obtain singular matrix.

3) We suppose now that the theorem holds for matrices of type $(n-1) \times 3$ and $3 \times(n-1)$, where $n-1>3$.

4) If we take now matrix $A=[A]_{n \times 3}$ and $B=[B]_{3 \times n}$ and try to calculate the determinant of the product $A B$, we can do it with minor expansion choosing the last row in the matrix $A B$. Each thus obtained minor will be determinate of matrix that is result of multiplying matrices of type $(n-1) \times 3$ and $3 \times(n-1)$ and according to 3 ) all these minors have value 0 , which proves the theorem.

We can generalize these results and prove the next theorem.

Theorem 2.4. Multiplying matrix $A=[A]_{n \times k}$ with $B=[B]_{k \times n}$, for $n>k$, we always obtain as result singular $n \times n$ matrix.

Proof. We will prove first that the theorem holds for the product $[A B]_{k \times k}=$ $[A]_{k \times(k-1)} \cdot[B]_{(k-1) \times k}$ and then, using this result, we will prove the general case by induction regarding $n$. 


$$
\text { We take } A=\left[\begin{array}{cccc}
a_{11} & a_{12} & \ldots & a_{1(k-1)} \\
a_{21} & a_{22} & \ldots & a_{2(k-1)} \\
\ldots & \ldots & \ldots & \ldots \\
a_{k 1} & a_{k 2} & \ldots & a_{k(k-1)}
\end{array}\right] \text { and } B=\left[\begin{array}{cccc}
b_{11} & b_{12} & \ldots & b_{1 k} \\
b_{21} & b_{22} & \ldots & b_{2 k} \\
\ldots & \ldots & \ldots & \ldots \\
b_{(k-1) 1} & b_{(k-1) 2} & \ldots & b_{(k-1) k}
\end{array}\right] \text {. }
$$

The product $A B$ is

$$
A B=\left[\begin{array}{cccc}
\sum_{j=1}^{k-1} a_{1 j} b_{j 1} & \sum_{j=1}^{k-1} a_{1 j} b_{j 2} & \ldots & \sum_{j=1}^{k-1} a_{1 j} b_{j k} \\
\sum_{j=1}^{k-1} a_{2 j} b_{j 1} & \sum_{j=1}^{k-1} a_{2 j} b_{j 2} & \ldots & \sum_{j=1}^{k-1} a_{2 j} b_{j k} \\
\ldots & \ldots & \ldots & \ldots \\
\sum_{j=1}^{k-1} a_{k j} b_{j 1} & \sum_{j=1}^{k-1} a_{k j} b_{j 2} & \ldots & \sum_{j=1}^{k-1} a_{k j} b_{j k}
\end{array}\right]
$$

Let us now compute the determinant of the last matrix. We will multiply each row except the first one with $\frac{1}{a_{11}} \cdot a_{11}$ and then obtain:

$$
\operatorname{det} A B=\frac{1}{a_{11} k-1}\left|\begin{array}{cccc}
\sum_{j=1}^{k-1} a_{1 j} b_{j 1} & \sum_{j=1}^{k-1} a_{1 j} b_{j 2} & \ldots & \sum_{j=1}^{k-1} a_{1 j} b_{j k} \\
\sum_{j=1}^{k-1} a_{11} a_{2 j} b_{j 1} & \sum_{j=1}^{k-1} a_{11} a_{2 j} b_{j 2} & \ldots & \sum_{j=1}^{k-1} a_{11} a_{2 j} b_{j k} \\
\ldots & \ldots & \ldots & \ldots \\
\sum_{j=1}^{k-1} a_{11} a_{k j} b_{j 1} & \sum_{j=1}^{k-1} a_{11} a_{k j} b_{j 2} & \ldots & \sum_{j=1}^{k-1} a_{11} a_{k j} b_{j k}
\end{array}\right|
$$

We will add the first row multiplied by $\left(-a_{j 1}\right)$ to the $j-$ th row, for $j=2,3, \ldots k$, i.e. add the first row multiplied by $\left(-a_{21}\right)$ to the second row; add the first row multiplied by $\left(-a_{31}\right)$ to the third row, etc. We will obtain:

$$
\operatorname{det} A B=\frac{1}{a_{11} k-1}\left|\begin{array}{cccc}
\sum_{j=1}^{k-1} a_{1 j} b_{j 1} & \sum_{j=1}^{k-1} a_{1 j} b_{j 2} & \ldots & \sum_{j=1}^{k-1} a_{1 j} b_{j k} \\
\sum_{j=2}^{k-1} b_{j 1}\left(a_{11} a_{2 j}-a_{21} a_{1 j}\right) & \sum_{j=2}^{k-1} b_{j 2}\left(a_{11} a_{2 j}-a_{21} a_{1 j}\right) & \ldots & \sum_{j=1}^{k-1} b_{j k}\left(a_{11} a_{2 j}-a_{21} a_{1 j}\right) \\
\ldots & \ldots & \ldots & \ldots \\
\sum_{j=2}^{k-1} b_{j 1}\left(a_{11} a_{k j}-a_{j 1} a_{1 j}\right) & \sum_{j=2}^{k-1} b_{j 2}\left(a_{11} a_{k j}-a_{j 1} a_{1 j}\right) & \ldots & \sum_{j=2}^{k-1} b_{j k}\left(a_{11} a_{k j}-a_{j 1} a_{1 j}\right)
\end{array}\right|
$$


For simplification of the notation, we will substitute the terms in the small brackets: we will denote $A_{i j}^{1}=a_{11} a_{i j}-a_{i 1} a_{1 j}$, i.e. $A_{2 j}^{1}=a_{11} a_{2 j}-a_{21} a_{1 j}, A_{3 j}^{1}=$ $a_{11} a_{3 j}-a_{31} a_{1 j}$, etc. Applying this substitution in the $\operatorname{det} A B$, we have:

$$
\operatorname{det} A B=\frac{1}{a_{11}{ }^{k-1}}\left|\begin{array}{cccc}
\sum_{j=1}^{k-1} a_{1 j} b_{j 1} & \sum_{j=1}^{k-1} a_{1 j} b_{j 2} & \cdots & \sum_{j=1}^{k-1} a_{1 j} b_{j k} \\
\sum_{j=2}^{k-1} b_{j 1} A_{2 j}^{1} & \sum_{j=2}^{k-1} b_{j 2} A_{2 j}^{1} & \cdots & \sum_{j=1}^{k-1} b_{j k} A_{2 j}^{1} \\
\cdots & \cdots & \cdots & \cdots \\
\sum_{j=2}^{k-1} b_{j 1} A_{k j}^{1} & \sum_{j=2}^{k-1} b_{j 2} A_{k j}^{1} & \cdots & \sum_{j=2}^{k-1} b_{j k} A_{k j}^{1}
\end{array}\right|
$$

We will consider $A_{22}^{1}$ as multiplier of the second row, $A_{32}^{1}$ as multiplier of the third row, etc. and consider $A_{k 2}^{1}$ as multiplier of the last row. Getting each of them as multiplier of the determinant, we obtain:

$\operatorname{det} A B=\frac{A_{22}^{1} A_{32}^{1} \cdots A_{k 2}^{1}}{a_{11} k-1}\left|\begin{array}{cccc}\sum_{j=1}^{k-1} a_{1 j} b_{j 1} & \sum_{j=1}^{k-1} a_{1 j} b_{j 2} & \ldots & \sum_{j=1}^{k-1} a_{1 j} b_{j k} \\ b_{21}+\sum_{j=3}^{k-1} b_{j 1} \frac{A_{2 j}^{1}}{A_{22}^{1}} & b_{22}+\sum_{j=3}^{k-1} b_{j 2} \frac{A_{2 j}^{1}}{A_{22}^{1}} & \ldots & b_{22}+\sum_{j=3}^{k-1} b_{j k} \frac{A_{2 j}^{1}}{A_{22}^{1}} \\ \ldots & \ldots & \ldots & \ldots \\ b_{21}+\sum_{j=3}^{k-1} b_{j 1} \frac{A_{k j}^{1}}{A_{k 2}^{1}} & b_{22}+\sum_{j=3}^{k-1} b_{j 2} \frac{A_{k j}^{1}}{A_{k 2}^{1}} & \ldots & b_{22}+\sum_{j=3}^{k-1} b_{j k} \frac{A_{k j}^{1}}{A_{k 2}^{1}}\end{array}\right|$

Multiplying the second row with $(-1)$ and adding to all rows bellow it, we have:

$$
\operatorname{det} A B=\frac{A_{22}^{1} A_{32}^{1} \cdots A_{k 2}^{1}}{a_{11} k-1}\left|\begin{array}{cccc}
\sum_{j=1}^{k-1} a_{1 j} b_{j 1} & \sum_{j=1}^{k-1} a_{1 j} b_{j 2} & \ldots & \sum_{j=1}^{k-1} a_{1 j} b_{j k} \\
b_{21}+\sum_{j=3}^{k-1} b_{j 1} \frac{A_{2 j}^{1}}{A_{22}^{1}} & b_{22}+\sum_{j=3}^{k-1} b_{j 2} \frac{A_{2 j}^{1}}{A_{22}^{1}} & \ldots & b_{22}+\sum_{j=3}^{k-1} b_{j k} \frac{A_{2 j}^{1}}{A_{22}^{1}} \\
\sum_{j=3}^{k-1} b_{j 1}\left(\frac{A_{3 j}^{1}}{A_{32}^{1}}-\frac{A_{2 j}^{1}}{A_{22}^{1}}\right) & \sum_{j=3}^{k-1} b_{j 2}\left(\frac{A_{3 j}^{1}}{A_{32}^{1}}-\frac{A_{2 j}^{1}}{A_{22}^{1}}\right) & \ldots & \sum_{j=3}^{k-1} b_{j k}\left(\frac{A_{3 j}^{1}}{A_{32}^{1}}-\frac{A_{2 j}^{1}}{A_{22}^{1}}\right) \\
\sum_{j=3}^{k-1} b_{j 1}\left(\frac{A_{k j}^{1}}{A_{k 2}^{1}}-\frac{A_{2 j}^{1}}{A_{22}^{1}}\right) & \sum_{j=3}^{k-1} b_{j 2}\left(\frac{A_{k j}^{1}}{A_{k 2}^{1}}-\frac{A_{2 j}^{1}}{A_{22}^{1}}\right) & \ldots & \sum_{j=3}^{k-1} b_{j k}\left(\frac{A_{k j}^{1}}{A_{k 2}^{1}}-\frac{A_{2 j}^{1}}{A_{22}^{1}}\right)
\end{array}\right|
$$


For simplification in the notation, we will substitute again the terms in the small brackets. We will denote: $A_{i j}^{2}=\frac{A_{i j}^{1}}{A_{i 2}^{1}}-\frac{A_{2 j}^{1}}{A_{22}^{1}}$ and then have:

$\operatorname{det} A B=\frac{A_{22}^{1} A_{32}^{1} \cdots A_{k 2}^{1}}{a_{11}^{k-1}}$

$$
\begin{array}{cccc}
\sum_{j=1}^{k-1} a_{1 j} b_{j 1} & \sum_{j=1}^{k-1} a_{1 j} b_{j 2} & \ldots & \sum_{j=1}^{k-1} a_{1 j} b_{j k} \\
b_{21}+\sum_{j=3}^{k-1} b_{j 1} \frac{A_{2 j}^{1}}{A_{22}^{1}} & b_{22}+\sum_{j=3}^{k-1} b_{j 2} \frac{A_{2 j}^{1}}{A_{22}^{1}} & \ldots & b_{22}+\sum_{j=3}^{k-1} b_{j k} \frac{A_{2 j}^{1}}{A_{22}^{1}}
\end{array}
$$

$$
\begin{array}{llll}
\sum_{j=3}^{k-1} b_{j 1} A_{3 j}^{2} & \sum_{j=3}^{k-1} b_{j 2} A_{3 j}^{2} & \ldots & \sum_{j=3}^{k-1} b_{j k} A_{3 j}^{2} \\
\sum_{j=3}^{k-1} b_{j 1} A_{k j}^{2} & \sum_{j=3}^{k-1} b_{j 2} A_{k j}^{2} & \ldots & \sum_{j=3}^{k-1} b_{j k} A_{k j}^{2}
\end{array}
$$

Similar as above, we will consider $A_{33}^{2}$ as multiplier of the second row, $A_{43}^{2}$ as multiplier of the third row, etc. and consider $A_{k 3}^{2}$ as multiplier of the last row. Getting again each of them as multiplier of the determinant, we obtain:

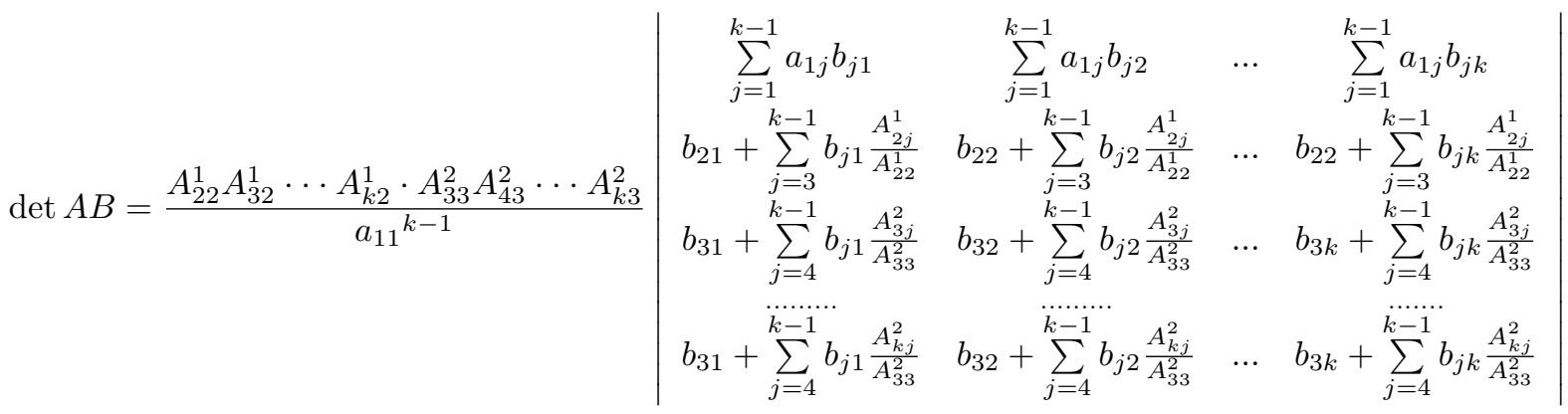

In the next step, we will add the third row multiplied with $(-1)$ to all the rows bellow it and thus obtain:

$$
\begin{aligned}
& \operatorname{det} A B= \\
& \frac{A_{22}^{1} A_{32}^{1} \cdots A_{k 2}^{1} \cdot A_{33}^{2} A_{43}^{2} \cdots A_{k 3}^{2}}{a_{11}{ }^{k-1}} \times \\
& \begin{array}{llll}
\sum_{j=1}^{k-1} a_{1 j} b_{j 1} & \sum_{j=1}^{k-1} a_{1 j} b_{j 2} & \ldots & \sum_{j=1}^{k-1} a_{1 j} b_{j k}
\end{array} \\
& b_{21}+\sum_{j=3}^{k-1} b_{j 1} \frac{A_{2 j}^{1}}{A_{22}^{1}} \quad b_{22}+\sum_{j=3}^{k-1} b_{j 2} \frac{A_{2 j}^{1}}{A_{22}^{1}} \quad \ldots \quad b_{2 k}+\sum_{j=3}^{k-1} b_{j k} \frac{A_{2 j}^{1}}{A_{22}^{1}} \\
& b_{31}+\sum_{j=4}^{k-1} b_{j 1} \frac{A_{3 j}^{2}}{A_{33}^{2}} \quad b_{32}+\sum_{j=4}^{k-1} b_{j 2} \frac{A_{3 j}^{2}}{A_{33}^{2}} \quad \ldots \quad b_{3 k}+\sum_{j=4}^{k-1} b_{j k} \frac{A_{3 j}^{2}}{A_{33}^{2}} \\
& \sum_{j=4}^{k-1} b_{j 1}\left(\frac{A_{4 j}^{2}}{A_{43}^{2}}-\frac{A_{3 j}^{2}}{A_{33}^{2}}\right) \quad \sum_{j=4}^{k-1} b_{j 2}\left(\frac{A_{4 j}^{2}}{A_{43}^{2}}-\frac{A_{3 j}^{2}}{A_{33}^{2}}\right) \quad \ldots \quad \sum_{j=4}^{k-1} b_{j k}\left(\frac{A_{4 j}^{2}}{A_{43}^{2}}-\frac{A_{3 j}^{2}}{A_{33}^{2}}\right) \\
& \begin{array}{cccc}
\cdots & \cdots & \cdots & \cdots \\
\sum_{j=4}^{k-1} b_{j 1}\left(\frac{A_{k j}^{2}}{A_{k 3}^{2}}-\frac{A_{3 j}^{2}}{A_{33}^{2}}\right) & \sum_{j=4}^{k-1} b_{j 2}\left(\frac{A_{k j}^{2}}{A_{k 3}^{2}}-\frac{A_{3 j}^{2}}{A_{33}^{2}}\right) & \cdots & \sum_{j=4}^{k-1} b_{j k}\left(\frac{A_{k j}^{2}}{A_{k 3}^{2}}-\frac{A_{3 j}^{2}}{A_{33}^{2}}\right)
\end{array}
\end{aligned}
$$


We will denote $A_{i j}^{3}=\frac{A_{i j}^{2}}{A_{i 3}^{2}}-\frac{A_{3 j}^{2}}{A_{33}^{2}}, i=4, \ldots k$, to simplify the notations (to write in a short way the terms in the brackets) and repeat previous steps starting from the fourth row: we will consider $A_{4 j}^{3}$ as multiplier of the fourth row, etc. consider $A_{k j}^{3}$ as multiplier of the last row, then get them as multipliers of the determinant. Then we will add the fourth row multiplied with $(-1)$ to the rows bellow it, etc. We can notice that when we add certain row multiplied by $(-1)$ to the others bellow, in the sums in those rows bellow, we eliminate one element, so the sums bellow have one element less. After finite number of steps, we will obtain:

$$
\begin{aligned}
& \operatorname{det} A B=\frac{\prod_{j=2}^{k} A_{j 2}^{1} \prod_{j=3}^{k} A_{j 3}^{2} \prod_{j=4}^{k} A_{j 4}^{3} \cdots \prod_{j=k-2}^{k} A_{i(k-2)}^{k-3}}{a_{11}{ }^{k-1}} \times \\
& \times \begin{array}{cccc}
\sum_{j=1}^{k-1} a_{1 j} b_{j 1} & \sum_{j=1}^{k-1} a_{1 j} b_{j 2} & \ldots & \sum_{j=1}^{k-1} a_{1 j} b_{j k} \\
b_{21}+\sum_{j=3}^{k-1} b_{j 1} \frac{A_{2 j}^{1}}{A_{22}^{1}} & b_{22}+\sum_{j=3}^{k-1} b_{j 2} \frac{A_{2 j}^{1}}{A_{22}^{1}} & \ldots & b_{22}+\sum_{j=3}^{k-1} b_{j k} \frac{A_{2 j}^{1}}{A_{22}^{1}} \\
\ldots & \ldots & \ldots & \ldots \\
b_{(k-2) 1}+b_{(k-1) 1} \frac{A_{(k-2)(k-1)}^{k-3}}{A_{(k-2)(k-2)}^{k-3}} & b_{(k-2) 2}+b_{(k-1) 2} \frac{A_{(k-2)(k-1)}^{k-3}}{A_{(k-2)(k-2)}^{k-3}} & \ldots & b_{(k-2) k}+b_{(k-1) k} \frac{A_{(k-2)(k-1)}^{k-3}}{A_{(k-2)(k-2)}^{k-3}} \\
b_{(k-2) 1}+b_{(k-1) 1} \frac{A_{(k-1)(k-1)}^{k-3}}{A_{(k-1)(k-2)}^{k-3}} & b_{(k-2) 2}+b_{(k-1) 2} \frac{A_{(k-1)(k-1)}^{k-3}}{A_{(k-1)(k-2)}^{k-3}} & \ldots & b_{(k-2) k}+b_{(k-1) k} \frac{A_{(k-1)(k-1)}^{k-3}}{A_{(k-1)(k-2)}^{k-3}} \\
b_{(k-2) 1}+b_{(k-1) 1} \frac{A_{k(k-1)}^{k-3}}{A_{k(k-2)}^{k-3}} & b_{(k-2) 2}+b_{(k-1) 2} \frac{A_{k(k-1)}^{k-3}}{A_{k(k-2)}^{k-3}} & \ldots & b_{(k-2) k}+b_{(k-1) k} \frac{A_{k(k-1)}^{k-3}}{A_{k(k-2)}^{k-3}}
\end{array}
\end{aligned}
$$

Now we will add the $(k-2)$ - th row multiplied with $(-1)$ to the last two row and thus obtain:

$$
\begin{aligned}
& \operatorname{det} A B=\frac{\prod_{j=2}^{k} A_{j 2}^{1} \prod_{j=3}^{k} A_{j 3}^{2} \prod_{j=4}^{k} A_{j 4}^{3} \ldots \prod_{j=k-2}^{k} A_{i(k-2)}^{k-3}}{a_{11}{ }^{k-1}} \times \\
& \times \begin{array}{cccc}
\sum_{j=1}^{k-1} a_{1 j} b_{j 1} & \sum_{j=1}^{k-1} a_{1 j} b_{j 2} & \ldots & \sum_{j=1}^{k-1} a_{1 j} b_{j k} \\
b_{21}+\sum_{j=3}^{k-1} b_{j 1} \frac{A_{2 j}^{1}}{A_{22}^{1}} & b_{22}+\sum_{j=3}^{k-1} b_{j 2} \frac{A_{2 j}^{1}}{A_{22}^{1}} & \ldots & b_{22}+\sum_{j=3}^{k-1} b_{j k} \frac{A_{2 j}^{1}}{A_{22}^{1}} \\
\cdots & \ldots & \ldots & \ldots \\
b_{(k-2) 1}+b_{(k-1)} \frac{A_{(k-2)(k-1)}^{k-3}}{A_{(k-2)(k-2)}^{k-3}} & \ldots & b_{(k-2) k}+b_{(k-1) k} \frac{A_{(k-2)(k-1)}^{k-3}}{A_{(k-2)(k-2)}^{k-3}} \\
b_{(k-1) 1}\left(\frac{A_{(k-1)(k-1)}^{k-3}}{\left.A_{(k-1)(k-2)}^{k-3}-\frac{A_{(k-2)(k-1)}^{k-3}}{A_{(k-2)(k-2)}^{k-3}}\right)}\right. & \cdots & b_{(k-1) k}\left(\frac{A_{(k-1)(k-1)}^{k-3}}{\left.A_{(k-1)(k-2)}^{k-3}-\frac{A_{(k-2)(k-1)}^{k-3}}{A_{(k-2)(k-2)}^{k-3}}\right)}\right. \\
b_{(k-1) 1}\left(\frac{A_{k(k-1)}^{k-3}}{\left.A_{k(k-2)}^{k-3}-\frac{A_{(k-2)(k-1)}^{k-3}}{A_{(k-2)(k-2)}^{k-3}}\right)}\right. & \cdots & b_{(k-1) k}\left(\frac{\left.A_{k(k-1)}^{k-3}-\frac{A_{(k-2)(k-1)}^{k-3}}{A_{k(k-2)}^{k-3}}\right)}{A_{(k-2)(k-2)}^{k-3}}\right)
\end{array} \mid
\end{aligned}
$$


Using substitution $A_{(k-1)(k-1)}^{k-2}=\frac{A_{(k-1)(k-1)}^{k-3}}{A_{(k-1)(k-2)}^{k-3}}-\frac{A_{(k-2)(k-1)}^{k-3}}{A_{(k-2)(k-2)}^{k-3}}$ and $A_{k(k-1)}^{k-2}=\frac{A_{k(k-1)}^{k-3}}{A_{k(k-2)}^{k-3}}-$ $\frac{A_{(k-2)(k-1)}^{k-3}}{A_{(k-2)(k-2)}^{k-3}}$ we have:

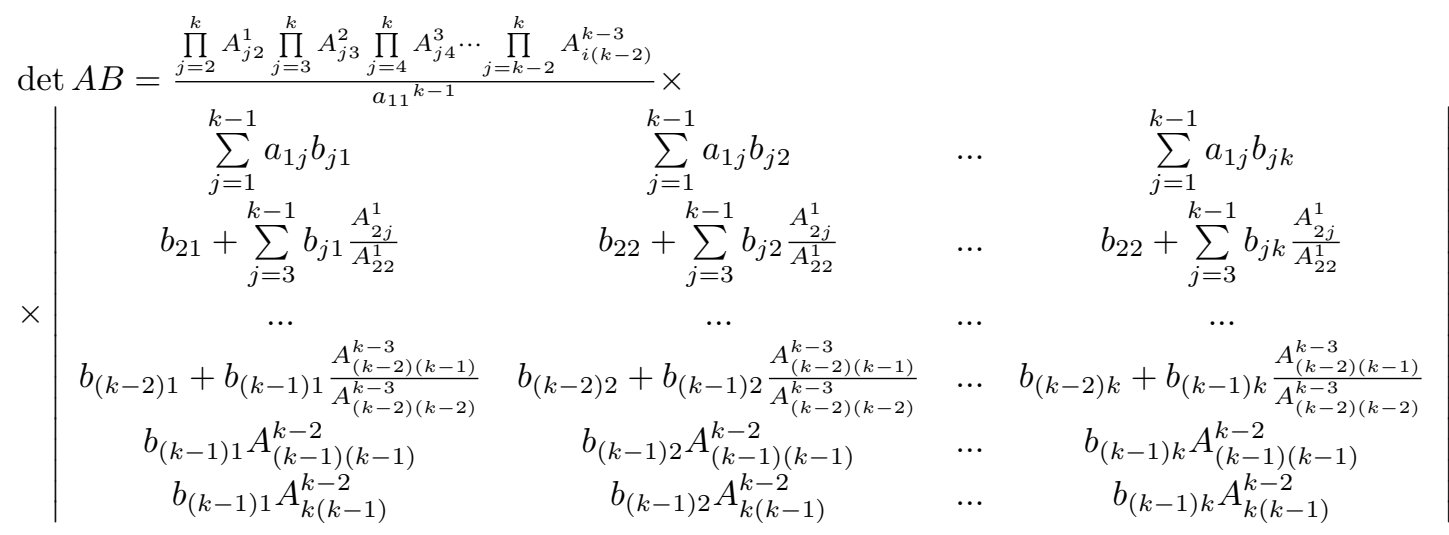

Finally, getting $A_{(k-1)(k-1)}^{k-2}$ and $A_{k(k-1)}^{k-2}$ from the last two rows as multipliers of the determinant, we obtain:

$$
\begin{aligned}
& \operatorname{det} A B=\frac{\prod_{j=2}^{k} A_{j 2}^{1} \prod_{j=3}^{k} A_{j 3}^{2} \prod_{j=4}^{k} A_{j 4}^{3} \ldots \prod_{j=k-2}^{k} A_{j(k-2)}^{k-3} \prod_{j=k-1}^{k} A_{j(k-1)}^{k-2}}{a_{11}-1} \\
& \times \begin{array}{cccc}
k-1 \\
\sum_{j=1}^{k-1} a_{1 j} b_{j 1} & \sum_{j=1}^{k-1} a_{1 j} b_{j 2} & \ldots & \sum_{j=1}^{k-1} a_{1 j} b_{j k} \\
b_{21}+\sum_{j=3}^{k-1} b_{j 1} \frac{A_{2 j}^{1}}{A_{22}^{1}} & b_{22}+\sum_{j=3}^{k-1} b_{j 2} \frac{A_{2 j}^{1}}{A_{22}^{1}} & \ldots & b_{22}+\sum_{j=3}^{k-1} b_{j k} \frac{A_{2 j}^{1}}{A_{22}^{1}} \\
\ldots & \ldots & \ldots & \ldots \\
b_{(k-2) 1}+b_{(k-1) 1} \frac{A_{(k-2)(k-1)}^{k-3}}{A_{(k-2)(k-2)}^{k-3}} & b_{(k-2) 2}+b_{(k-1) 2} \frac{A_{(k-2)(k-1)}^{k-3}}{A_{(k-2)(k-2)}^{k-3}} & \ldots & b_{(k-2) k}+b_{(k-1) k} \frac{A_{(k-2)(k-1)}^{k-3}}{A_{(k-2)(k-2)}^{k-3}} \\
b_{(k-1) 1} & b_{(k-1) 2} & \ldots & b_{(k-1) k} \\
b_{(k-1) 1} & b_{(k-1) 2} & \ldots & b_{(k-1) k}
\end{array}
\end{aligned}
$$

The determinant has value 0 as it has two equal rows. So, multiplying $k \times$ $(k-1)$ matrix with $(k-1) \times k$ matrix, we obtain singular $k \times k$ matrix.

The proof that the Theorem 2.4 holds for the product $A B$ of arbitrary matrices $A=[A]_{n \times k}$ and $B=[B]_{k \times n}$, where $n>k$, i.e. the product $A B$ in this case is singular $n \times n$ matrix, can easily be done by induction. We have already proved that the theorem holds when $n=k+1$. We suppose that the theorem holds for $(n-1)$, where $n-1>k$, and using this we can prove that it holds for all $n>k$. 


$$
\begin{gathered}
\text { If we take } A=\left[\begin{array}{cccc}
a_{11} & a_{12} & \ldots & a_{1 k} \\
a_{21} & a_{22} & \ldots & a_{2 k} \\
\ldots & \ldots & \ldots & \ldots \\
a_{n 1} & a_{n 2} & \ldots & a_{n k}
\end{array}\right] \text { and } B=\left[\begin{array}{cccc}
b_{11} & b_{12} & \ldots & b_{1 n} \\
b_{21} & b_{22} & \ldots & b_{2 n} \\
\ldots & \ldots & \ldots & \ldots \\
b_{k 1} & b_{k 2} & \ldots & b_{k n}
\end{array}\right] \text {, then } \\
\qquad A B=\left[\begin{array}{ccccc}
\sum_{j=1}^{k} a_{1 j} b_{j 1} & \sum_{j=1}^{k} a_{1 j} b_{j 2} & \ldots & \sum_{j=1}^{k} a_{1 j} b_{j n} \\
\sum_{j=1}^{k} a_{2 j} b_{j 1} & \sum_{j=1}^{k} a_{2 j} b_{j 2} & \ldots & \sum_{j=1}^{k} a_{2 j} b_{j n} \\
\ldots & \ldots & \ldots & \ldots \\
\sum_{j=1}^{k} a_{n j} b_{j 1} & \sum_{j=1}^{k} a_{n j} b_{j 2} & \ldots & \sum_{j=1}^{k} a_{n j} b_{j n}
\end{array}\right]
\end{gathered}
$$

To calculate the determinant of the last matrix, we can use minor expansion, for example, choosing the last row. Similar as in the previous theorems, each minor thus we obtain can be consider as determinant of matrix which is result of multiplication of $(n-1) \times k$ matrix with $k \times(n-1)$ matrix. According to the inductive assumption, all these minors have value 0 , so $\operatorname{det} A B=0$, which proves the theorem.

\section{CONCLUSION}

In this manuscript we have proved how we can obtain singular matrix by multiplying two randomly chosen matrices with specific form. Having in mind that singular matrices have big application in mathematics and other sciences, and also having in mind that the probability to generate randomly singular matrix is very low, we think that this paper will have big impact in mathematics, computational and other sciences which deal with matrices because the main result in the paper is exactly a way to generate singular matrix, and thus many scientists will have interest for this paper.

\section{REFERENCES}

[1] L. ElDEN: Matrix Methods in Data Mining and Pattern Recognition, The Society for Industrial and Applied Mathematics, 2007. 
[2] X. Wang, E. Serpedin: An Overview on the Applications of Matrix Theory in Wireless Communications and Signal Processing, Algorithms, 9(68) (2016), 1-21.

[3] D. S. YAdAV, R. K. Sharma, W. ShuKLA: On Applications of Singular Matrices over Finite Fields in Cryptography, InfoSecHiComNet, Security Aspects in Information Technology, (2011), 181-185.

[4] K. G. BRock: How rare are singular matrices?, The Mathematical Gazette, 89(516) (2005), 378-384.

[5] M. D. EATON, M. D. Perlman: The nonsingularity of generalized sample covariances matrices, Ann. Statist., 1(1973), 710-717.

[6] A. E. TAYLOR: Introduction to functional analysis, 2nd ed., Wiley, 1980.

[7] L. LovASZ: Singular spaces of matrices and their application in combinatorics, Bol. Soc. Braz. Mat., 20(1989), 87-99.

[8] G.N. Chen: On Some Properties of Singular Matrices, Linear Algebra and its Applications, 49(1983), 137-151.

[9] A. RANUM: The Group-Membership of Singular Matrices, American Journal of Mathematics, 31(1) (1909), 18-41.

[10] J. Kahn, J. Komlos, E. Szemeredi: On the Probability That a Random \pm 1 -Matrix Is Singular, Journal of the American Mathematical Society, 8(1) (1995), 223-240.

[11] J. Bourgain, V. H. VU, P. M. Wood: On the singularity probability of discrete random matrices, Journal of Functional Analysis 258(2010), 559-603.

Department of MATHEMATICS AND STATISTics

FACULTY OF COMPUTER SCIENCE

UNIVERSity GoCE DELCEV - STIP, MACEDONIA

E-mail address: marija.miteva@ugd.edu.mk

DEPARTMENT OF MATHEMATICS AND STATISTICS

FACULTY OF COMPUTER SCIENCE

UNIVERSity GoCE DELCEV - STIP, MACEDONIA

E-mail address: limonka. lazarova@ugd.edu.mk

FACULTY OF COMPUTER SCIENCE

University Goce DelCeV - Stip, MACEDonia

E-mail address: natasa.stojkovik@ugd.edu.mk

FACULTY OF COMPUTER SCIENCE

University Goce DelCeV - Stip, Macedonia

E-mail address: aleksandra.stojanova@ugd.edu.mk 